\title{
Comunicação
}

[Communication]

\section{Freqüência de aglutininas anti-Leptospira interrogans em eqüídeos, em Minas Gerais, 2003 a 2004}

[Frequency of anti-Leptospira interrogans agglutinins in equidae in Minas Gerais, Brazil, from 2003 to 2004]

\author{
D. Chiareli ${ }^{1}$, E.C. Moreira ${ }^{{ }^{*}}$, H.O.D. Gutiérrez ${ }^{1}$, R.O. Rodrigues ${ }^{1}$, A.P. Marcelino ${ }^{1}$, \\ J.N.C. Meneses ${ }^{1}$, V.M.A. Almeida ${ }^{2}$ \\ ${ }^{1}$ Escola de Veterinária - UFMG \\ Caixa Postal 567 \\ 30123-970 - Belo Horizonte, MG \\ ${ }^{2}$ Instituto Mineiro de Agropecuária - Belo Horizonte, MG
}

\begin{abstract}
As leptospiroses são enfermidades transmissíveis entre animais e o homem, amplamente difundidas e caracterizadas como doença febril aguda. Representam importantes zoonoses que causam problemas na saúde pública e prejuízo na criação de animais de produção. A maioria das infecções por leptospira é subclínica, e a principal fonte de infecção é um animal infectado, que contamina o pasto, a água e os alimentos via urina. Os animais contaminados podem eliminar leptospiras por longo período, mesmo após a cura clínica. A porta de entrada do microrganismo ocorre através da mucosa ocular, digestiva, respiratória e genital, ou até mesmo pela pele íntegra quando em contato, por longo período, com água contaminada (Fainne et al., 1999).
\end{abstract}

O objetivo do presente estudo foi determinar a freqüência e a distribuição espacial da infecção por Leptospira interrogans em eqüídeos em Minas Gerais de setembro de 2003 a março de 2004.

Foram analisadas 6475 amostras de soros sangüíneos de eqüídeos cedidas pelo Instituto Mineiro de Agropecuária (IMA), coletados no período de setembro de 2003 a março de 2004, em Minas Gerais. Esses soros vieram de propriedades que possuíam eqüídeos de serviço com baixo valor zootécnico e sem raça definida.
A idade mínima estudada foi de animais acima de seis meses para que não houvesse a interferência dos anticorpos colostrais. $\mathrm{O}$ número de propriedades amostradas pelo IMA foi determinado de maneira aleatória, o que permitiu que os municípios com maior número de propriedades tivesse, proporcionalmente, maior participação. Para cada um dos estratos de amostragem, utilizou-se uma lista de produtores rurais ordenados por município de maneira aleatória e sistemática. Dessa maneira, todos os 853 municípios foram amostrados.

Para representar a distribuição espacial da infecção por Leptospira interrogans nos eqüídeos, as 12 mesorregiões de Minas Gerais foram agrupadas em sete estratos: 1- Norte de Minas/Noroeste de Minas, 2- Vale do Mucuri/Jequitinhonha, 3- Vale do Rio Doce, 4Central Mineira/Oeste de Minas/Metropolitana de Belo Horizonte, 5- Sul/Sudoeste de Minas, 6Triângulo Mineiro/Alto Paranaíba e 7- Campos das Vertentes/Zona da Mata.

As análises foram realizadas pela técnica de microaglutinação rápida (MAR), descrita por Ryu (1970). As sorovariedades de Leptospira spp. utilizadas como antígenos no MAR foram culturas vivas de Bratislava, Ballum, Batavie, Canicola, Copenhageni, Icterohaemorrhagiae, Pomona e Hardjo (Norma). Essas amostras 
foram obtidas da Organização Pan-americana da Saúde e uma delas, L. interrogans sorovariedade Hardjo (Norma), foi isolada por Moreira (1994) em bovinos em Minas Gerais. A tipificação final foi feita no Royal Tropical Institute em Amsterdã, Holanda. A reação de MAR foi positiva quando ocorreu, no mínimo, $50 \%$ de aglutinação, em diluições iguais ou superiores a $1: 200$.

Utilizaram-se planilhas eletrônicas do programa Microsoft Excel. Na base de dados, cada amostra de soro sangüíneo indicou o município de origem, o estrato ou mesorregião, a data de coleta, a idade, a raça, o sexo, a espécie e as sorovariedades utilizadas na bateria de antígenos, resultado das reações de microaglutinação, e o título das reações.

Das 6.475 amostras de soro de eqüídeos analisadas, 381 apresentaram reações positivas, com títulos iguais ou acima de 1:200 para uma ou mais sorovariedades de leptospiras, representando $5,9 \%$ dos eqüídeos positivos.

As sorovaridades de Leptospira interrogans mais freqüentes foram Hardjo (Norma) $(3,0 \%)$ e Pomona (1,2\%), seguidas de Bratislava $(0,66 \%)$, Batavie $\quad(0,53 \%), \quad$ Canicola $\quad(0,42 \%)$, Icterohaemorrhagiae $(0,42 \%)$, Ballum $(0,06 \%) \mathrm{e}$ Copenhageni $(0,02 \%)$. Não era esperado que a Hardjo (Norma) fosse a mais freqüente das sorovariedades encontradas em eqüídeos, pois são os bovinos os hospedeiros de manutenção dessa sorovariedade (Ellis et al., 1981; Araujo et al., 2005), sendo, por isso, mais bem adaptada a essa espécie. Os eqüídeos, nesse caso, seriam seu hospedeiro acidental. Entretanto, em Minas Gerais, a maioria dos eqüídeos é criada no mesmo hábitat, dividindo pastagens $\mathrm{e}$ bebedouros com bovinos, e, desse modo, o contato direto ou indireto entre as espécies justificaria esse resultado. Pellegrin et al. (1994) também encontraram alta freqüência de animais positivos para hardjo, confirmando que a infecção ocorre por serem os bovinos os hospedeiros de manutenção e, dessa forma, contaminariam o ambiente e transmitiriam a infecção para os eqüinos.

Títulos positivos para sorovariedade Pomona já foram relatados no Brasil por Barbosa (1962), em Minas Gerais; Santa Rosa et al. (1968), em São Paulo; Cordeiro et al. (1974), em Minas
Gerais; Pinheiro et al. (1985), em eqüídeos procedentes dos estados de Goiás, Minas Gerais, Mato Grosso do Sul, Paraná e São Paulo; Abuchaim (1991), no Rio Grande do Sul; Lilenbaum (1998) no Rio de Janeiro, e Linhares et al. (2005), em Goiás. Santa Rosa et al. (1968), ao correlacionarem positividade dos eqüinos para essa sorovariedade e presença de oftalmia periódica que evoluía para cegueira, observaram que em quase $50 \%$ dos casos a enfermidade envolvia os dois olhos. Donahue et al. (1995), correlacionarem sua positividade com casos de aborto eqüino, nos Estados Unidos, isolaram a sorovariedade Kennewicki do sorogrupo pomona, em 43 casos e Pomona em uma amostra. Bovinos e suínos são os hospedeiros mais adaptados à Pomona; os eqüinos, no entanto, são hospedeiros acidentais dessa sorovariedade, o que explica a patogenicidade elevada para essa espécie (Lilenbaum,1998; Langoni et al., 2004).

Ellis et al. (1983), na Irlanda do Norte, sugeriram que os eqüinos são os hospedeiros de manutenção da Bratislava. No Brasil, essa sorovariedade foi encontrada por Lilenbaum (1998), em eqüinos no Rio de Janeiro, 27,2\% reagentes. No Rio Grande do Sul, foi a sorovariedade mais freqüente verificada por Oliveira e Pires Neto (2004), 20,5\% de eqüinos reagentes. Os testes sorológicos não são considerados suficientes para concluir sobre a presença de uma sorovaridade especifica na região ou mesmo no país. É necessário que se faça o isolamento e posterior tipificação de leptospira devido à ocorrência de reações sorológicas cruzadas, como ressaltam Fainne et al. (1999).

As sorovariedades Canicola e Icterohaemorrhagiae foram encontradas em baixas porcentagens em eqüídeos, em Minas Gerais. A Canicola tem no cão seu hospedeiro natural, sendo encontrada em maior freqüência nessa espécie (Magalhães et al., 2006), enquanto os roedores são os hospedeiros naturais da Icterohaemorrhagiae. Tais sorovariedades são mais comuns em eqüídeos que vivem próximos às áreas urbanas, devido à maior possibilidade de contato com seus respectivos hospedeiros naturais. Provavelmente, os animais dessa pesquisa tiveram pouco contato com essas leptospiras devido ao fato de habitarem ambientes rurais. 
Titulações maiores igual a 1:25600 para Pomona, Bratislava, Bataviae, Canicola e Icterohaemorrhagiae (Tab. 1), sugerem que essas sorovariedades estão presentes em Minas Gerais, e seria necessário o isolamento e a tipificação para confirmação. Além disso, os altos títulos encontrados, principalmente aqueles iguais ou maiores que 1:400, evidenciam que houve contato recente com essas leptospiras, o que poderia, em alguns casos, evoluir para uma doença grave.

Tabela 1. Distribuição dos títulos positivos de soro de eqüídeos avaliados por meio da técnica de microaglutinação rápida em sorovariedades de Leptospira interrogans, Minas Gerais, 2003

\begin{tabular}{|c|c|c|c|c|c|c|c|c|c|c|c|c|c|c|c|c|}
\hline \multirow{3}{*}{ Sorovariedade } & \multicolumn{16}{|c|}{ Título } \\
\hline & \multicolumn{2}{|c|}{$1: 200$} & \multicolumn{2}{|c|}{$1: 400$} & \multicolumn{2}{|c|}{$1: 800$} & \multicolumn{2}{|c|}{$1: 1600$} & \multicolumn{2}{|c|}{$1: 3200$} & \multicolumn{2}{|c|}{$1: 6400$} & \multicolumn{2}{|c|}{$1: 12800$} & \multicolumn{2}{|c|}{$\geq 1: 25600$} \\
\hline & $\%$ & $\mathrm{P}$ & $\%$ & $\mathrm{P}$ & $\%$ & $\mathrm{P}$ & $\%$ & $\mathrm{P}$ & $\%$ & $\mathrm{P}$ & $\%$ & $\mathrm{P}$ & $\%$ & $\mathrm{P}$ & $\%$ & $\mathrm{P}$ \\
\hline Hardjo & 27,6 & 54 & 13,8 & 27 & 14,8 & 29 & 6,1 & 12 & 6,6 & 13 & 1,5 & 3 & 3,1 & 6 & 26,5 & 52 \\
\hline Pomona & 28,8 & 23 & 16,3 & 13 & 26,3 & 21 & 3,7 & 3 & 3,7 & 3 & 5,0 & 4 & 3,7 & 3 & 12,5 & 10 \\
\hline Bratislava & 32,5 & 14 & 14,0 & 6 & 9,2 & 4 & 2,3 & 1 & - & 0 & 7,0 & 3 & - & 0 & 35,0 & 15 \\
\hline Bataviae & 44,1 & 15 & 8,8 & 3 & 20,6 & 7 & 2,9 & 1 & - & 0 & - & 0 & - & 0 & 23,6 & 8 \\
\hline Canicola & 14,8 & 4 & 18,5 & 5 & 14,8 & 4 & 25,9 & 7 & 3,7 & 1 & 7,4 & 2 & 3,7 & 1 & 11,2 & 3 \\
\hline Ictero & 55,6 & 15 & 18,5 & 5 & 11,1 & 3 & 3,7 & 1 & - & 0 & - & 0 & 3,7 & 1 & 7,4 & 2 \\
\hline ballum & 50,0 & 2 & 25,0 & 1 & - & 0 & - & 0 & 25,0 & 1 & - & 0 & - & 0 & - & 0 \\
\hline Copenhageni & 100,0 & 1 & - & 0 & - & 0 & - & 0 & - & 0 & - & 0 & - & 0 & - & 0 \\
\hline
\end{tabular}

$\mathrm{P}=$ positivo

O estrato Norte/Nordeste de Minas apresentou a maior freqüência, seguido pelos estratos Triângulo Mineiro/Alto Paranaíba, Central Mineira/Oeste de Minas/Metropolitana de Belo
Horizonte e Sul/Sudoeste de Minas. Vale do Rio Doce foi o estrato com menor porcentagem de animais positivos (Tab. 2).

Tabela 2. Freqüência de Aglutininas anti-Leptospira interrogans em soros sangüíneos de equídeos, por estrato, Minas Gerais, 2003-2004

\begin{tabular}{|c|c|c|c|c|c|c|}
\hline \multirow{2}{*}{\multicolumn{2}{|c|}{ Estrato }} & \multirow{2}{*}{ Amostra } & \multicolumn{2}{|c|}{ Resultado } & \multirow[t]{2}{*}{ Freqüência \% } & \multirow{2}{*}{$\begin{array}{c}\text { Intervalo de } \\
\text { Confiança }\end{array}$} \\
\hline & & & Negativo & Positivo & & \\
\hline Norte de Minas/Noroeste de Minas & & 1066 & 980 & 86 & 8,07 & $6,5-9,9$ \\
\hline Triângulo Mineiro/Alto Paranaíba & & 698 & 648 & 50 & 7,16 & $5,4-9,3$ \\
\hline $\begin{array}{l}\text { Central Mineira/Oeste de } \\
\text { Metropolitana de Belo Horizonte }\end{array}$ & Minas/ & 909 & 849 & 60 & 6,60 & $5,1-8,4$ \\
\hline Sul/Sudoeste de Minas & & 778 & 731 & 47 & 6,04 & $4,5-7,9$ \\
\hline Campo das Vertentes/Zona da Mata & & 701 & 666 & 35 & 4,99 & $3,5-6,9$ \\
\hline Vale do Mucuri/Jequitinhonha & & 1424 & 1360 & 64 & 4,49 & 3,5 \\
\hline Vale do Rio Doce & & 899 & 860 & 39 & 4,34 & $3,1-5,9$ \\
\hline Total & & 6475 & 6094 & 381 & 5,88 & $5,3-6,5$ \\
\hline
\end{tabular}

Triângulo Mineiro/Alto Paranaíba, Norte/Noroeste de Minas, Central Mineira/Oeste de Minas/Metropolitana de Belo Horizonte e Sul/Sudoeste de Minas são estratos que apresentam elevado efetivo bovino. São semelhantes em relação ao perfil de produção agropecuária, com propriedades empresariais e tecnologicamente atualizadas. Possuem acesso a assistência veterinária e, por isso, teriam grande chance de instituir programas sanitários para prevenção e controle de leptospiroses. Seria importante investigar nesses estratos qual o papel epidemiológico dos eqüídeos na manutenção da sorovariedade Hardjo, pois há possibilidade de esses animais contribuírem para a continuidade dessa infecção nos bovinos, mesmo após tentativas de controle.

Os resultados obtidos na presente pesquisa revelam que, provavelmente, as boas condições de criação de eqüídeos para serviço em Minas Gerais foram determinantes para a baixa freqüência de animais reagentes para leptospira.

Palavras-chave: eqüideos, Leptospira, freqüência, distribuição espacial 


\begin{abstract}
The goal of this research was to find the frequency and spatial distribution of infection caused by Leptospira interrogans in equidae in Minas Gerais State from September 2003 to March 2004. Samples of blood serum $(6,475)$ were analyzed by microscopic agglutination test. From the total, 381 samples were positive $(5.9 \%)$, with title equal or superior to 1:200 for one or more serovars of leptospira. The most frequent serovars were Hardjo (Norma), Pomona, Bratislava, and Batavie. The higher frequency of equidae reagents were recorded at the North and Northeast region of Minas Gerais, followed by Triângulo Mineiro and Alto Paranaiba, then Central, West, Metropolitan area of Belo Horizonte, and South/Southwest.
\end{abstract}

Keywords: equidae, Leptospira, frequency, spatial distribution

\section{REFERÊNCIAS BIBLIOGRÁFICAS}

ABUCHAIM, D.M. Presença de aglutininas antileptospira em soro de eqüinos no Estado do Rio Grande do Sul. Arq. Fac. Vet. UFRGS, v.19, p.9-14, 1991.

ARAUJO, V.E.M.; MOREIRA, E.C.; NAVEDA, L.A.B. et al. Freqüência de aglutininas anti-Leptospira interrogans em soros sangüíneos de bovinos, em Minas Gerais, de 1980 a 2002. Arq. Bras. Med. Vet. Zootec., v.57, p.430-435, 2005.

BARBOSA M. Aglutininas e lisinas antileptospira em soros de bovinos, eqüinos e suínos em Minas Gerais. Arq. Esc. Vet. UFMG, v.14, p.1-26, 1962.

CORDEIRO, P.; RAMOS A.A.; BATISTA JÚNIOR J.A. Aglutininas antileptospira em soro eqüino em Minas Gerais. Rev. Agropec. Bras., v.9, p.45-48, 1974.

DONAHUE, J.M.; SMITH, B.J.; POONACHA, K.B. et al. Prevalence and serovars of leptospira involved in equine abortions in central Kentucky during the 19911993 foaling seasons. J. Vet. Diagn. Invest., v.7, p.8791, 1995.

ELLIS, W.A.; O`BRIEN, J.J.; CASSELLS J.A. et al. Leptospiral infection in horses in Northern Ireland: serological and microbiological findings. Equine Vet. J., v.15, p.317-320, 1983.

ELLIS, W.A.; O'BRIEN, J.J.; CASSELLS, J. Role of cattle in the maintenance of Leptospira interrogans serotype hardjo infection in North Ireland. Vet. Rec., v.108, p.555-557, 1981.

FAINE, S.; ADLER, B.; BOLIN, C. et al. Leptospira and leptospiroses. 2.ed. Sydney: MediSci, 1999. 272p.

LANGONI, H.; DA SILVA, A.V.; PEZERICO, S.B. et al. Anti-leptospirose agglutinins in equine sera, from São Paulo, Goiás, and Mato Grosso do Sul, Brazil, 1996-2001. J. Venom. Anim. Toxins Trop. Dis., v.10, p.207-208, 2004.
LILENBAUM W. Leptospirosis on animal reproduction: IV. Serological findings in mares from six farms in Rio de Janeiro, Brazil (1993- 1996). Braz. J. Vet. Res. Anim. Sci., v.35, p.61-63, 1998.

LINHARES, G.F.C.; GIRIO, R.J.S.; LINHARES, D.C.L. et al. Sorovariedades de Leptospira interrogans e respectivas prevalências em cavalos da Microrregião de Goiânia, GO. Cienc. Anim. Bras., v.6, p.255-259, 2005.

MAGALHAES, D.F.; SILVA, J.A.; MOREIRA, E.C. et al. Prevalência de aglutininas anti-Leptospira interrogans em cães de Belo Horizonte, Minas Gerais, 2001 a 2002. Arq. Bras. Med. Vet. Zootec., v.58, p.167-17. 2006.

MOREIRA, E.C. Avaliação de métodos para erradicação de leptospiroses em bovinos. 1994. 94f. Tese (Doutorado) - Escola de Veterinária, Universidade Federal de Minas Gerais, Belo Horizonte.

OLIVEIRA, S.J.; PIRES NETO, J.A.S. Aspectos etiológicos e diagnóstico nas leptospiroses. Rev. CFMV, v.10, p.36-46, 2004.

PELLEGRIN, A.O.; SILVA, R.A.M.S.; RIBEIRO, S.C. Ocorrência de aglutininas anti-Leptospira em eqüinos do Pantanal Mato-Grossense, sub-região da Nhecolândia. In: CONGRESSO BRASILEIRO DE MEDICINA VETERINÁRIA, 23., 1994, Olinda. Anais... Olinda: SPEMV, 1994. p.190.

PINHEIRO, H.H.; SILVEIRA, W.; OLIVEIRA, V.C. Pesquisa de aglutininas antileptospira em soros de eqüinos abatidos no Frigorífico Xavante - Araguari, MG. A Hora Vet., v.5, p.42-43, 1985.

RYU, E. Rapid microscopic agglutination test for Leptospira without nonspecific reaction. Bull. Off. Int. Epizoot., v.73, p.49-58, 1970.

SANTA ROSA, C.A.; CASTRO A.F.P.D.; CAMPEDELLI FILHO O. et al. Leptospirose em eqüinos. Arq. Inst. Biol., v.35, p.61-65, 1968. 